\begin{tabular}{|c|c|}
\hline Title & A n LMS A daptive A rray for Multipath-Fading Reduction \\
\hline Author(s) & Ogawa, Y asutaka; Ohmiya, Manabu; Itoh, Kiyohiko \\
\hline Citation & IEEE Transactions on A erospace and Electronic Systems, 23(1), 17-23 \\
\hline Issue Date & $1987-01$ \\
\hline Doc URL & http:/hdl .handle.net/2115/5963 \\
\hline Rights & $\begin{array}{l}\text { O1987 IEEE. Personal use of this material is permitted. However, permission to reprint/republish this material for } \\
\text { advertising or promotional purposes or for creating new collective works for resale or redistribution to servers or lists, } \\
\text { or to reuse any copyrighted component of this work in other works must be obtained from the IEEE." } \\
\text { IEEE, IEEE Transactions on A erospace and Electronic Systems } \\
\text {,23(1), 1987, p17-23 }\end{array}$ \\
\hline Tyре & article \\
\hline File Information & ITAES23_1.pdf \\
\hline
\end{tabular}

Instructions for use 


\section{An LMS Adaptive Array for Multipath Fading Reduction}

\author{
YASUTAKA OGAWA, Member, IEEE \\ MANABU OHMIYA \\ KIYOHIKO ITOH, Member, IEEE \\ Hokkaido University
}

\begin{abstract}
Multipath fading often poses a serious hindrance in radio . communication. The application of a least-mean-square (LMS) adaptive array to the problem of multipath fading reduction is discussed. However, it is known that multipath components are in general correlated with one another. We examine the effect of the correlation on the performance of the LMS adaptive array. When the correlation coefficient does not equal or approximate 1 , the LMS adaptive array suppresses the multipath signals significantly by nulling. On the other hand, when the correlation coefficient nearly equals 1 , the LMS adaptive array prevents the output signal power from decreasing. Therefore, the LMS adaptive array may reduce the multipath fading effectively for any correlation coefficient value. A reference signal in the LMS adaptive array is also discussed. It is shown that synchronization in the reference signal generation must be extremely accurate. Moreover, a processor configuration is proposed which may generate the reference signal with the required accuracy.
\end{abstract}

Manuscript received September 11, 1985; revised March 29, 1986.

Authors' address: Department of Electronic Engineering, Faculty of Engineering, Hokkaido University, Kita 13, Nishi 8, Kita-ku, Sapporo 060, Japan.

0018-9251/87/0100-0017\$1.00 이 1987 IEEE

\section{INTRODUCTION}

Radio communications suffer from multipath fading. It has been reported that only a few multipath components are often dominant in strength and play an important role in the multipath fading phenomena [1]. Thus, an adaptive array [2] has a potential to reduce the multipath fading.

A least-mean-square (LMS) adaptive array automatically tracks a desired signal and nulls interference signals. The LMS adaptive array, however, requires a reference signal in order to control each weight. Let us assume that the desired signal contains a deterministic component which is fully known at the receiver. Then, the deterministic component may be used for the adaptive array reference signal. An example of the deterministic component is a pilot signal which is added to the transmitted communication signal $[3,6]$. According to the literature [3], the LMS adaptive array may eliminate the undesired multipath signals by nulling, in a case where a modulated pilot signal has a sufficient bandwidth to discriminate between multipath propagation modes. This means that the LMS adaptive array may suppress the undesired multipath components when the incident components are not correlated with one another. In mobile communications, however, we do not know the time delay differences between multipath components. Then, the required bandwidth of the pilot signal is in general unknown. Even though we know the time delay differences, an unrealistically wide bandwidth might be required. Thus, in order to reduce the multipath fading by the adaptive array, we must consider the effect of the correlation between multipath components on the performance of the adaptive array.

The literature [4] proposed a preprocessing scheme for the adaptive array which may suppress the coherent signals. A disadvantage of this scheme is that it needs more antenna elements than the conventional adaptive array.

We show that an LMS adaptive array may reduce the multipath fading effectively for any correlation coefficient value between multipath signals. First, we examine the behavior of the LMS adaptive array in the presence of the correlated multipath signals. Second, we find required synchronization accuracy in reference signal generation. Third, we propose a processor configuration which generates the reference signal.

\section{FORMULATION OF THE PROBLEM}

We consider the $\mathrm{N}$-element linear LMS adaptive array shown in Fig. 1. We assume that two multipath components $\tilde{s}(t)$ and $\tilde{m}(t)$ are incident on the array from angles $\theta_{s}$ and $\theta_{m}$ relative to broadside, respectively. The antenna elements are assumed to be isotropic and a halfwavelength apart. We represent both signals on the $k$ th element by $\tilde{s}_{k}(t)$ and $\tilde{m}_{k}(t)(k=1 \sim N)$. Thermal noise $\tilde{n}_{k}(t)$ is assumed to be present on each element signal. 


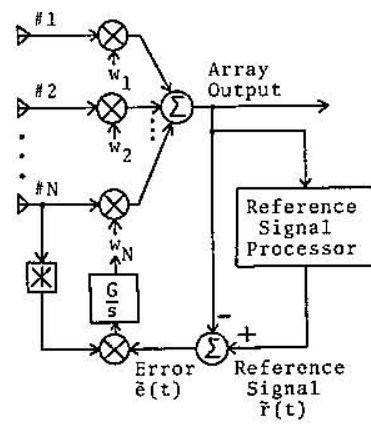

Fig. 1. LMS adaptive array.

Then, the complex-valued element signal is given by

$\tilde{x}_{k}(t)=\tilde{s}_{k}(t)+\tilde{m}_{k}(t)+\tilde{n}_{k}(t)$.

We assume that the thermal noise components on different elements are independent and that they are also independent of the signals $\tilde{s}(t)$ and $\tilde{m}(t)$.

We define an $\mathrm{N}$-dimensional signal vector as

$X(t)=\left[\tilde{x}_{1}(t) \tilde{x}_{2}(t) \ldots \tilde{x}_{N}(t)\right]^{\mathrm{T}}$

where $\mathrm{T}$ denotes transpose. Furthermore, we express the complex weight for the $k$ th element as $w_{k}$ and the $N$ dimensional weight vector as $W$, i.e.,

$W=\left[w_{1} w_{2} \ldots \bar{w}_{N}\right]^{\mathrm{T}}$.

We assume that the parameter $G$ in Fig. 1 is determined adequately so that the deviation of the weight vector $W$ from the ensemble average is negligibly small. Thus, we consider the weight vector $W$ to have the ensemble average.

We represent the power of $\tilde{s}_{k}(t), \bar{m}_{k}(t)$, and $\tilde{n}_{k}(t)$ by $S_{i}, M_{i}$, and $N_{i}$, respectively. Namely, we have

$S_{i}=\left\langle\left|\tilde{s}_{k}(t)\right|^{2}\right\rangle, \quad M_{i}=\left\langle\left|\tilde{m}_{k}(t)\right|^{2}\right\rangle$

$N_{i}=\left\langle\left|\tilde{n}_{k}(t)\right|^{2}\right\rangle \quad$ for $k=1 \sim N$

where $\langle\cdot\rangle$ denotes the ensemble average.

It is assumed that $\tilde{m}_{1}(t)$ is delayed from $\tilde{s}_{1}(t)$ by $\tau$. Then, $\tilde{m}_{1}(t)$ is expressed as

$\tilde{m}_{1}(t)=\sqrt{M_{i} / S_{i}} \tilde{s}_{1}(t-\tau) e^{-j \Psi^{\prime}}$

where $\Psi^{\prime}$ is a phase delay which occurs by a reason other than the propagation delay difference.

In order to generate a reference signal $\tilde{r}(t)$ in the LMS adaptive array, a pilot signal is always transmitted together with an information signal $[3,6]$. The power spectrum of the signal is illustrated in Fig. 2. The pilot signal is modulated by a signal which is fully known at the receiver. Both bands are located very closely to each other. Namely, let $\omega_{c}$ and $\omega_{c}^{\prime}$ be the center angular frequencies of the pilot and information portions, respectively. Then, $\left|\omega_{c}-\omega_{c}^{\prime}\right| / \omega_{c}<<1$ holds. The reference signal which is generated by the reference signal processor is a replica of the pilot portion of the signal. We assume that the bandwidth of the pilot signal

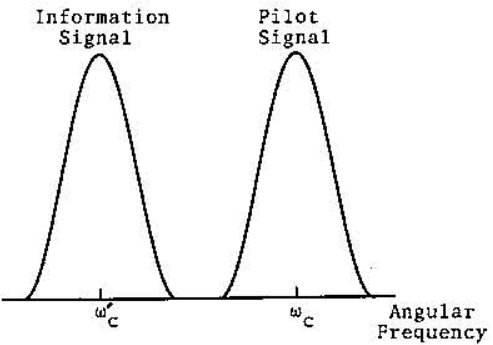

Fig. 2. Spectrum of signal.

is almost the same as that of the information signal. For simplicity, we consider only the pilot portion.

We express the reference signal as

$\tilde{r}(t)=\tilde{s}_{1}(t-T r)$.

When $\operatorname{Tr}=0$, the reference signal coincides with $\tilde{s}(t)$. Similarly, when $T r=\tau$, it coincides with $\tilde{m}(t)$.

Now, we represent the complex envelopes of $\tilde{s}_{1}(t)$ and $\tilde{m}_{1}(t)$ by $\tilde{S}_{1}(t)$ and $\tilde{M}_{1}(t)$, respectively. Then, we have

$\tilde{s}_{1}(t)=\tilde{S}_{1}(t) e^{j \omega_{c} t}$

$\tilde{m}_{1}(t)=\tilde{M}_{1}(t) e^{j \omega_{c} t}$.

From (5), (7), (8), we may obtain

$\tilde{M}_{1}(t)=\sqrt{M_{i} / S_{i}} \tilde{S}_{1}(t-\tau) e^{-j\left(\omega_{c} \tau+\Psi^{\prime}\right)}$.

We define the normalized autocorrelation function of $\tilde{S}_{1}(t)$ as

$\tilde{\rho}\left(t^{\prime}\right)=\left\langle\tilde{S}_{1}^{*}(t) \tilde{S}_{1}\left(t+t^{\prime}\right)\right\rangle / S_{i}$

where $*$ denotes the complex conjugate.

Moreover, we define the covariance matrix $R x x$ and correlation vector $V x r$ as

$R x x=\left\langle X^{*}(t) X^{\mathrm{T}}(t)\right\rangle$

$V x r=\left\langle X^{*}(t) \tilde{r}(t)\right\rangle$.

Here, we assume that the bandwidth of the pilot signal is narrow enough that the interelement delay does not change the envelope.

From these results, the $(p, q)$ th element of $R x x$ is given by

$$
\begin{aligned}
& \left\langle\tilde{x}_{p}^{*}(t) \tilde{x}_{q}(t)\right\rangle \\
& =S_{i} e^{j(p-q) \phi_{s}}+M_{i} e^{j(p-q) \phi_{m}}+N_{i} \delta_{p q} \\
& \quad+\sqrt{S_{i} M_{i}} \tilde{\rho}^{*}(\tau) e^{j\left((p-1) \phi_{s}-(q-1) \phi_{m}-\omega_{c} \tau-\Psi^{\prime}\right\}} \\
& \quad+\sqrt{S_{i} M_{i}} \tilde{\rho}(\tau) e^{\left.j(p-1) \phi_{m}-(q-1) \phi_{s}+\omega_{c} \tau+\Psi^{\prime}\right\}}
\end{aligned}
$$

where

$\phi_{s}=\pi \sin \theta_{s}$

$\phi_{m}=\pi \sin \theta_{m}$

and $\delta_{p q}$ is the Kronecker's $\delta$. 
Similarly, the $k$ th element of $V x r$ is given by

$$
\begin{aligned}
& \left\langle\tilde{x}_{k}^{*}(t) \tilde{r}(t)\right\rangle \\
& =S_{i} \tilde{\rho}^{*}(T r) e^{j\left\{(k-1) \phi_{s}-\omega_{c} T r\right\}} \\
& \quad+\sqrt{S_{i} M_{i}} \tilde{\rho}(\tau-T r) e^{\left.j(k-1) \phi_{m}+\omega_{c} \tau+\Psi^{\prime}-\omega_{c} T r\right\}} .
\end{aligned}
$$

Here, it should be noted that the following equation holds.

$$
\left\langle\tilde{s}_{1}^{*}(t) \tilde{m}_{1}(t)\right\rangle / \sqrt{S_{i} M_{i}}=\tilde{\rho}^{*}(\tau) e^{-j\left(\omega_{c} \tau+\Psi^{\prime}\right)}
$$

This means that $\tilde{\rho}^{*}(\tau) e^{-j\left(\omega_{c} \tau+\Psi^{\prime}\right)}$ is the complexvalued correlation coefficient between $\tilde{s}_{1}(t)$ and $\tilde{m}_{1}(t)$.

As stated previously, we consider that the weight vector has the ensemble average. Thus, the steady-state weight vector is given by the Wiener solution, i.e.,

$W=R x x^{-1} V x r$.

This is true whether the incident signals are correlated with or not.

We represent the array output of $\tilde{s}(t), \tilde{m}(t)$, and the thermal noise by $\tilde{s}_{0}(t), \tilde{m}_{0}(t)$, and $\tilde{n}_{0}(t)$, respectively. Moreover, we represent the power of them by $S_{0}, M_{0}$, and $N_{0}$. Namely, we have

$$
\begin{aligned}
S_{0} & =\left\langle\left|\tilde{s}_{0}(t)\right|^{2}\right\rangle / 2 ; \quad M_{0}=\left\langle\left|\tilde{m}_{0}(t)\right|^{2}\right\rangle / 2 \\
N_{0} & =\left\langle\left|\tilde{n}_{0}(t)\right|^{2}\right\rangle / 2 .
\end{aligned}
$$

When $S_{0} \geq M_{0}$, we consider that $\bar{s}(t)$ is the desired signal and $\tilde{m}(t)$ is the undesired one. On the other hand, when $M_{0}>S_{0}$, we consider that $\tilde{m}(t)$ is the desired signal and $\tilde{s}(t)$ is the undesired one. Here, we represent the desired-to-undesired-signal-ratio by DUR. Note that the output DUR is given by $S_{0} / M_{0}$ when $S_{0} \geq M_{0}$ and that it is given by $M_{0} / S_{0}$ when $M_{0}>S_{0}$. Moreover, we add that all of the numerical results which are shown later are computed values.

\section{MULTIPATH FADING REDUCTION}

Now we discuss the steady-state performance of the LMS adaptive array. In this section we assume that $T r=0$ holds. Namely, we assume that the reference signal coincides with $\tilde{s}(t)$.

In order to simplify the notation, we introduce the real-valued symbols $C(0 \leq C \leq 1)$ and $\Psi$ which satisfy (20).

$C e^{-j \Psi}=\tilde{\rho}^{*}(\tau) e^{-j\left(\omega_{c} \tau+\Psi^{\prime}\right)}$.

From (17), it is seen that $C$ and $\Psi$ are the magnitude and phase delay of the correlation coefficient of $s_{1}(t)$ and $\tilde{m}_{1}(t)$, respectively.

Fig. 3 shows the output DUR versus $C$ for several values of $\Psi$. Since $S_{0} \geq M_{0}$ holds for these parameters, $\tilde{s}(t)$ is the desired signal and $\tilde{m}(t)$ is the undesired one. It is seen that the output DUR depends on the correlation coefficient $\left(C e^{-j \Psi}\right)$. The undesired signal is, however, suppressed significantly by the LMS adaptive array

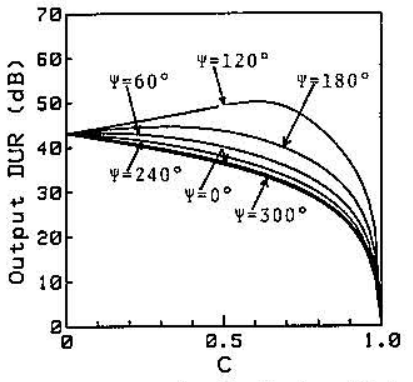

Fig. 3. Output DUR versus $C . N=2, \theta_{s}=0^{\circ}, \theta_{m}=30^{\circ}, T r=0$, $S_{\mathrm{i}} / N_{i}=M_{i} / N_{i}=20 \mathrm{~dB}$.

provided that $C$ does not equal or approximate 1 . The output DUR is above $20 \mathrm{~dB}$ when $C \leq 0.9$.

Fig. 4 illustrates the effect of $C$ on the array pattern. It is apparent that when $C \leq 0.9$, the null is pointed almost exactly toward $\tilde{m}(t)$. However, when $C>0.9$, the null is shifted or lost.

Now we discuss the case where $C$ nearly equals 1 . When $C \simeq 1, \tau$ is significantly less than the reciprocal of the frequency bandwidth of the signal. In this case, the fading is not frequency-selective but frequency-flat. Furthermore, we may regard $\tilde{d}_{0}^{\prime}(t)$ defined by (21) as the approximate output desired signal.

$\tilde{d}_{0}^{\prime}(t)=\tilde{s}_{0}(t)+\tilde{m}_{0}(t)$.

Let $D_{0}^{\prime}$ denote the power of $\tilde{d}_{0}^{\prime}(t)$, i.e.,

$D_{0}^{\prime}=\left\langle\left|\tilde{d}_{0}^{\prime}(t)\right|^{2}\right\rangle / 2$.

The output $\mathrm{D}^{\prime} \mathrm{NR}\left(D^{\prime}{ }_{0} / N_{0}\right)$ represents the approximate output desired-signal-to-noise-ratio when $C \simeq 1$.

Fig. 5 shows the output $\mathrm{D}^{\prime} \mathrm{NR}$ versus $C$ for several values of $\Psi$. From these curves, it is seen that the LMS adaptive array prevents the output signal power from decreasing. Namely, the frequency-flat fading is reduced. When $C \simeq 1$, the weights are determined in such a way

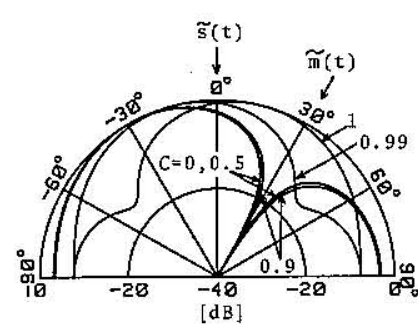

Fig. 4. Array pattern. $N=2, \theta_{s}=0^{\circ}, \theta_{m}=30^{\circ}, \Psi=0^{\circ}, T r=0$, $S_{i} / N_{i}=M_{i} / N_{i}=20 \mathrm{~dB}$.

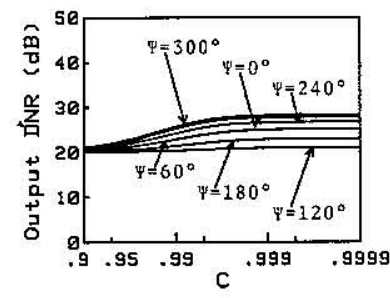

Fig. 5. Output $D^{\prime} N R$ versus $C . N=2, \theta_{s}=0^{\circ}, \theta_{m}=30^{\circ}, T r=0$, $S_{i} / N_{i}=M_{i} / N_{i}=20 \mathrm{~dB}$. 
that the weighted signals $w_{k}\left\{\tilde{s}_{k}(t)+\tilde{m}_{k}(t)\right\}(k=1 \sim N)$ are added in-phase at the array output. This means that the LMS adaptive array realizes space diversity when $C \simeq 1$.

Here we consider the physical reason why the weighted signals are added in-phase at the array output. As is shown later, the waveform distortion of the output signal component $\tilde{d}_{0}^{\prime}(t)=\sum_{k=1}^{N} w_{k}\left\{\tilde{s}_{k}(t)+\tilde{m}_{k}(t)\right\}$ is negligibly small. In other words, when $C \simeq 1$, the output signal component has almost the same waveform as that of the reference signal $\tilde{r}(t)=\tilde{s}_{1}(t)$. The LMS adaptive array shown in Fig. 1 realizes the weights which minimize the mean-square error $\left\langle|\bar{e}(t)|^{2}\right\rangle$. We see that the error is given by

$$
\begin{aligned}
\tilde{e}(t) & =\vec{r}(t)-\tilde{y}(t) \\
& =\tilde{s}_{1}(t)-\tilde{d}_{0}^{\prime}(t)-\sum_{k=1}^{N} w_{k} \tilde{n}_{k}(t) .
\end{aligned}
$$

The last term $\sum_{k=1}^{N} w_{k} \vec{n}_{k}(t)$ is the thermal noise. If the weighted signals are added in-phase at the array output, the output signal component $\tilde{d}_{0}^{\prime}(t)$ coinsides almost perfectly with the reference signal keeping the weight norm $\sqrt{W^{\dagger} W}$ small value ( $\dagger$ denotes complex conjugate transpose). This means that the thermal noise power $N_{i}$ $W^{\dagger} W$ in the error $\tilde{e}(t)$ has a lower value. Thus, the meansquare error is minimized by adding the weighted signals in-phase at the array output when $C \simeq 1$.

According to the literature [4], the signal cancellation phenomenon occurs when the desired signal is correlated with one or more interfering signals. When the incident signals are correlated with one another, the desired signal is canceled in adaptive arrays other than the LMS adaptive array shown in Fig. 1. Even though the incident signals are not correlated with one another, the desired signal may be canceled [7]. Under some circumstances, the weights do not converge to the Wiener solution. The "non-Wiener" effects cause signal cancellation [7]. However, as stated previously, the parameter $G$ in Fig. 1 is determined in such a way that the deviation of the weight vector from the ensemble average is negligibly small. Namely, the steady-state weight vector is given by the Wiener solution (18). Thus, the signal cancellation phenomenon due to the non-Wiener effects does not occur in the LMS adaptive array discussed in this paper. The LMS adaptive array does not cancel the desired signal even when the incident signals are correlated with one another. This is because the signal cancellation increases the mean-square error in the LMS adaptive array. It is shown analytically in the Appendix that the weighted signals $w_{k}\left\{\tilde{s}_{k}(t)+\tilde{m}_{k}(t)\right\}(k=1 \sim N)$ are added in-phase at the array output when $\tau=0(C=1)$ and that the signal cancellation phenomenon does not occur in the LMS adaptive array.

Moreover, we investigate the distortion contained in $\tilde{d}_{0}^{\prime}(t)$. We define the distortion power $E_{0}^{\prime}$ as

$E_{0}^{\prime}=\min _{\alpha}\left\langle\left|\tilde{d}_{0}^{\prime}(t)-\alpha \tilde{s}_{1}(t)\right|^{2}\right\rangle / 2$.
Fig. 6 shows the output $\mathrm{E}^{\prime} \mathrm{NR}\left(E_{0}^{\prime} / N_{0}\right)$ as a function of $C$ for several values of $\Psi$. We see that the output $\mathrm{E}^{\prime} \mathrm{NR}$ is less than $0 \mathrm{~dB}$. Namely, $E_{0}^{\prime}$ is less than the output thermal noise power. Therefore, we may say that although the multipath signal is not suppressed when $C \simeq$ 1 , the waveform distortion is negligible.

\section{REFERENCE SIGNAL GENERATION}

Thus far, $\operatorname{Tr}$ has been assumed to be 0 . Namely, we have assumed that the reference signal coincides exactly with $\bar{s}(t)$. In this section we discuss the problem of the reference signal generation. In the remainder of this paper, we assume that the pilot signal is biphase modulated by a pseudonoise ( $\mathrm{PN}$ ) sequence with a long period. Then, we express the normalized autocorrelation function $\tilde{\rho}(t)$ as

$\tilde{\rho}(t)=\left\{\begin{array}{cl}1-|t| / T, & \text { for }|t| \leq T \\ 0, & \text { elsewhere }\end{array}\right.$

where $T$ denotes a clock pulse duration.

A local PN sequence generator at the receiver modulates the carrier which is recovered from the array output. However, the reference signal in general does not coincide with the incident signal in time, i.e., $\operatorname{Tr} \neq 0$ and $\operatorname{Tr} \neq \tau$. Thus we must synchronize it to the time of arrival of $\tilde{s}(t)$ or $\tilde{m}(t)$.

\section{A. Synchronization Accuracy}

From the above assumptions, we examine the effect of $T r$ on the steady-state performance of the LMS adaptive array.

Fig. 7 shows the output DUR versus $T r / T$. When $\tau / T=0.5, \tilde{s}(t)$ and $\tilde{m}(t)$ are correlated with each other

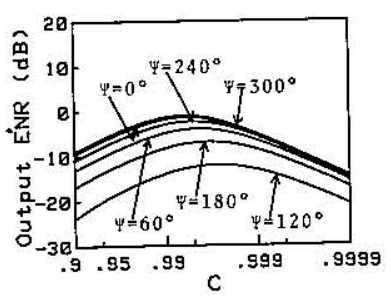

Fig. 6. Output $E^{\prime} \mathrm{NR}$ versus $C . N=2, \theta_{s}=0^{\circ}, \theta_{m}=30^{\circ}, T r=0$, $S_{i} / N_{i}=M_{i} / N_{i}=20 \mathrm{~dB}$.

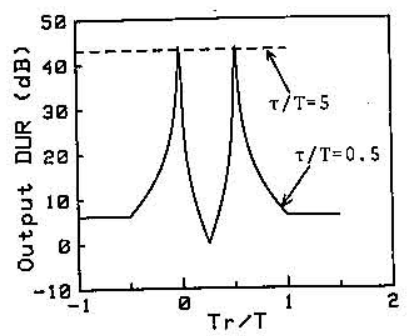

Fig. 7. Output DUR versus $\operatorname{Tr} / T . N=2, \theta_{s}=0^{\circ}, \theta_{m}=30^{\circ}, \Psi=0^{\circ}$, $S_{i} / N_{i}=M_{i} / N_{i}=20 \mathrm{~dB}$. 
according to (17) and (24). When $\tau / T=5$, they are independent of each other. When $T r / T=0$, the reference signal coincides with $\tilde{s}(t)$. Similarly, when $\tau / T=0.5$ and $T r / T=0.5$, it coincides with $\tilde{m}(t)$. It is seen that satisfactory output DUR is obtained around $T r / T=0$ or $T r / T=0.5$ for $\tau / T=0.5$. On the other hand, the output DUR has a steady and satisfactory value for $-1<T r / T$ $<1$ when $\tau / T=5$.

Fig. 8 shows each output power normalized by $N_{i} / 2$ versus $T r / T$ for $\tau / T=0.5$. The period for which the output undesired signal power is suppressed less than $N_{0}$ is about $0.2 T$ around $T r / T=0$ or $T r / T=0.5$.

From these results, we say that the synchronization of the reference signal must be extremely accurate in the case where the input multipath components are correlated with one another.

\section{B. Reference Signal Processor}

Now we propose the configuration of the reference signal processor. The reference signal generation consists of two parts just like the synchronization process in a spread spectrum receiver [5]. One is acquisition and the other is tracking. The acquisition is implemented by a sliding correlator [5] which performs the search process and calculates the correlation between the received pilot signal and reference signal. The sliding correlator makes the reference signal coincide with the pilot signal within $T$. During the initial acquisition, the reference signal is not correlated with the input signal and all of the weights are driven to 0 , if the LMS adaptive processor operates. Thus, until the initial acquisition is achieved, we do not make it operate. Namely, the weights are frozen, in fixed values, for example, $w_{1}=1, w_{2}=w_{3}=\ldots=w_{N}=0$.

Now we discuss the tracking process which is the second part of the synchronization. The tracking circuit operates in such a way that the reference signal coincides with the transmitted pilot signal as precisely as possible. When the multipath components are correlated with each other, the correlation function between the array output signal and generated reference signal is not a symmetric triangular function. Thus, a delay-lock loop [5] may not be employed. Also, it is difficult to achieve an accurate synchronization by use of the conventional tau-dither clock-tracking loop [5]. Thus; we must configure the new tracking circuit.

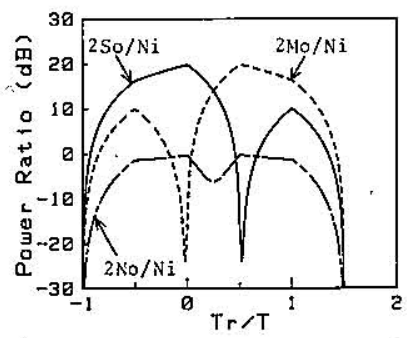

Fig. 8. Power ratio versus $T r / T . N=2, \theta_{s}=0^{\circ}, \theta_{m}=30^{\circ}, \Psi=0^{\circ}$, $\tau / T=0.5, S_{i} / N_{i}=M_{i} / N_{i}=20 \mathrm{~dB}$.
Fig. 9 shows the normalized MSE (mean-square error) in the LMS adaptive array versus $T r / T$. Here, the MSE is defined as

$\operatorname{MSE}=\left\langle|\tilde{e}(t)|^{2}\right\rangle$.

It is seen that when the reference signal coincides with $\tilde{s}(t)$ or $\tilde{m}(t)(T r / T=0$ or $T r / T=0.5)$, the MSE has an extremely low minimal value. Then, the MSE may be used for the recognition of the synchronization.

After the initial acquisition is achieved by the sliding correlator, we make the LMS adaptive processor and tracking circuit operate. The configuration of the tracking circuit is shown in Fig. 10. Note that each signal in Fig. 10 has a real value. The VCO (voltage-controlled oscillator) is controlled in such a way that the MSE has a minimal value. This circuit is analogous to the tau-dither clock-tracking loop used in a spread spectrum receiver. Fig. 11 shows an example of waveforms in the tracking circuit. We set the duration time $\left(T^{\prime}\right)$ of the rectangular wave $a(t)$ longer than the convergence time of the weights. The amplitude of $a(t)$ is $\sqrt{N_{i} / 2}$. Then, $a(t)=$ $\pm \sqrt{N_{i} / 2}$ holds. At a leading edge of $a(t)$, the clock phase of the reference signal is shifted back by a fraction $(\Delta T)$ of the clock pulse duration. It is shifted forth by the same amount at a trailing edge of $a(t)$. At the output

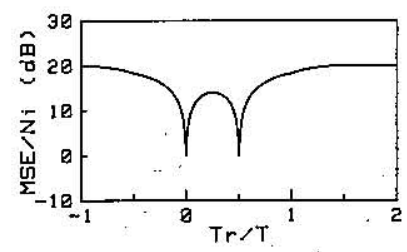

Fig. 9. MSE $/ N_{i}$ versus $T r / T . N=2, \theta_{s}=0^{\circ}, \theta_{m}=30^{\circ}, \Psi=0^{\circ}$, $\tau / T=0.5 S_{i} / N_{i}=M_{i} / N_{i}=20 \mathrm{~dB}$.

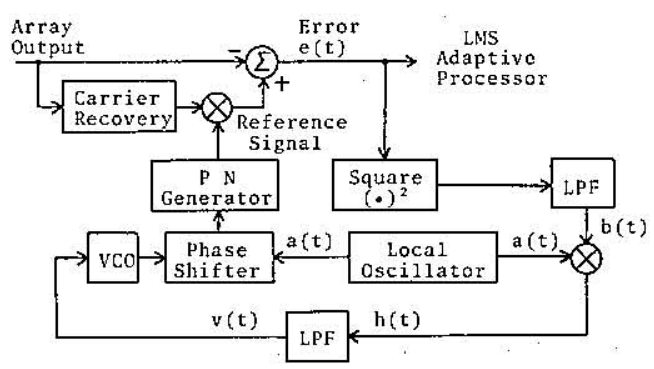

Fig. 10. Tracking circuit.

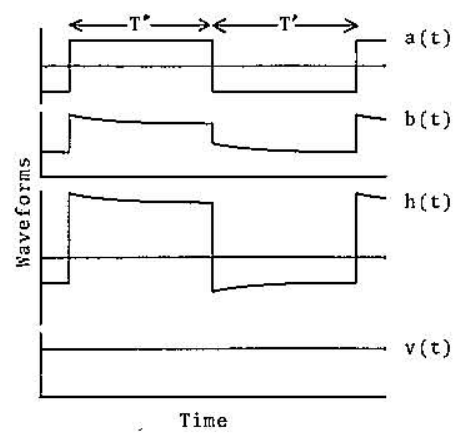

Fig. 11. Waveforms in tracking circuit. 
from the LPF (low-pass filter) placed behind the squaring device, we may obtain the waveform $b(t)$ which is almost proportional to the MSE $\left(\left\langle|\tilde{e}(t)|^{2}\right\rangle\right)$. We assume that $b(t)$ $=\sqrt{2}\left\langle|\hat{e}(t)|^{2}\right\rangle / \sqrt{N_{i}}$ holds. Then, we have $h(t)=$ $\pm\left\langle|\tilde{e}(t)|^{2}\right\rangle$. The LPF placed in front of the VCO extracts the DC component $v(t)$ from $h(t)$. Since the VCO is controlled by $v(t)$ in such a way that the MSE has a minimal value, the reference signal coincides with $\tilde{s}(t)$ or $\tilde{m}(t)$ in time. By using the tracking circuit, we may generate a highly accurate reference signal.

We assume that the transfer function of the LPF in front of the VCO is $K_{1} /\left(1+s T_{1}\right)$. Since the phase of the VCO-output signal is proportional to the integral of the control signal $v(t)$, we may have

$\frac{d}{d t}\left(\frac{T r}{T}\right)=K v(t)$

Fig. 12 and 13 show the variations of $T r / T$ and the output DUR, respectively, in a case where the tracking circuit operates. It is seen that $T r / T$ reaches the range from $-0.005(-\Delta T / 2 T)$ to $0.005(\Delta T / 2 T)$. This means that the reference signal is synchronized with the time of arrival of $\tilde{s}(t)$. It is also seen that the output DUR takes on satisfactory values when $T r / T$ approaches 0 . The output DUR ranges from $32.3 \mathrm{~dB}$ to $38.6 \mathrm{~dB}$ even after convergence. This is because $T r$ is vibrated.

\section{CONCLUSIONS}

We have examined the fading reduction performance of the LMS adaptive array. Moreover, we have discussed the problem of the reference signal generation and we have the following results.

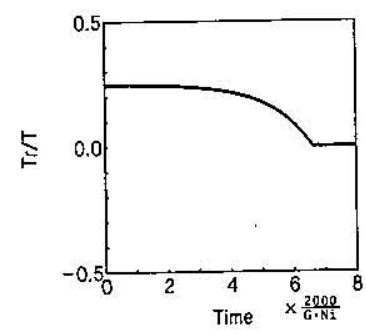

Fig. 12. $T r / T$ versus time. $N=2, \theta_{s}=0^{\circ}, \theta_{m}=30^{\circ}, \Psi=0^{\circ}$, $\tau / T=0.5, S_{i} / N_{i}=M_{i} / N_{i}=20 \mathrm{~dB}, \Delta T / T=0.01, T^{\prime}=10 /\left(G \cdot N_{i}\right)$,

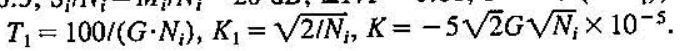

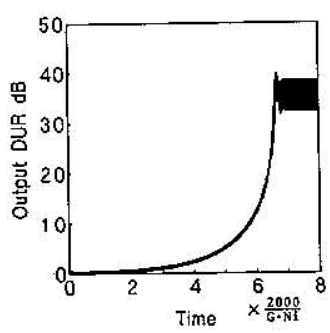

Fig. 13. Output DUR versus time. $N=2, \theta_{s}=0^{\circ}, \theta_{m}=30^{\circ}, \Psi=0^{\circ}$, $\tau / T=0.5, S_{i} / N_{i}=M_{i} / N_{i}=20 \mathrm{~dB}, \Delta T / T=0.01, T^{\prime}=10 /\left(G \cdot N_{i}\right)$ $T_{1}=100 /\left(G \cdot N_{i}\right), K_{1}=\sqrt{2 / N_{i}}, K=-5 \sqrt{2} G \sqrt{N_{i}} \times 10^{-5}$.
1) The behavior of the LMS adaptive array depends on the correlation coefficient of the incident signals. However, if the reference signal is generated properly, the LMS adaptive array may reduce the multipath fading effectively for any correlation coefficient value.

2) The synchronization in the reference signal generation must be very accurate in the case where the multipath components are correlated with one another.

3) We proposed a processor configuration which generates the reference signal. We showed satisfactory performance of the tracking circuit.

\section{APPENDIX. THEORETICAL ANALYSIS FOR} $\tau=0(C=1)$

We assume that $\tau=0$ holds. From (10) and (20), we obtain $C=1$. Namely, $\tilde{m}(t)$ is perfectly coherent with $\tilde{s}(t)$. In this case, the following equations hold.

$$
\begin{aligned}
\tilde{s}_{k}(t) & =\tilde{S}_{1}(t) e^{j\left\{\omega_{c} t-(k-1) \phi_{s}\right\}} \\
\tilde{m}_{k}(t) & =\tilde{M}_{1}(t) e^{j\left\{\omega_{c} t-(k-1) \phi_{m}\right\}} \\
& =\sqrt{M_{i} / S_{i}} \tilde{S}_{1}(t) e^{j\left\{\omega_{c} t-(k-1) \phi_{m}-\Psi^{\prime}\right\}} .
\end{aligned}
$$

Then, the combined signal on each antenna element is given by

$\tilde{s}_{k}(t)+\tilde{m}_{k}(t)=b_{k} \bar{S}_{1}(t) e^{j \omega_{c} t}, \quad(k=1 \sim N)$

where

$b_{k}=e^{-j(k-1) \phi_{s}}+\sqrt{M_{i} / S_{i}} e^{-j\left\{(k-1) \phi_{m}+\Psi^{\prime}\right\}}$.

We define an $N$-dimentional vector as

$B=\left[b_{1}, b_{2} \ldots, b_{N}\right]^{\mathrm{T}}$.

From these results, we have

$R x x=S_{i} B^{*} B^{\mathrm{T}}+N_{i} I$

where $I$ denotes an $N \times N$ identity matrix.

Also, assuming that $\operatorname{Tr}=0$ holds, we obtain

$V x r=S_{i} B^{*}$.

Even when the signals $\tilde{s}(t)$ and $\tilde{m}(t)$ are correlated with each other, the steady-state ensemble average of the weight vector is given by the Wiener solution $R x x^{-1} \mathrm{Vxr}$. Then, from (A6) and (A7), we may obtain

$$
\begin{aligned}
W & =\left(S_{i} B^{*} B^{\mathrm{T}}+N_{i} I\right)^{-1} S_{i} B^{*} \\
& =\frac{S_{i}}{S_{i} B^{\mathrm{T}} B^{*}+N_{i}} B^{*} .
\end{aligned}
$$

Furthermore, from (A3) and (A8), each weighted signal is given by

$$
\begin{aligned}
& w_{k}\left\{\tilde{s}_{k}(t)+\tilde{m}_{k}(t)\right\} \\
& =\frac{S_{i}\left|b_{k}\right|^{2}}{S_{i} B^{\mathrm{T}} B^{*}+N_{i}} \tilde{S}_{1}(t) e^{j \omega_{c} t}, \quad(k=1 \sim N) .
\end{aligned}
$$


We see that the phase of each weighted signal has the same value. Therefore, it may be said that the weighted signals are added in-phase at the array output and that the signal cancellation phenomenon does not occur.

\section{REFERENCES}

[1] Ikegami, F., and Yoshida, S. (1980)

Analysis of multipath propagation structure in urban mobile radio environments.

IEEE Transactions on Antennas and Propagation, AP-28, 4 (July 1980), 531-537.

[2] Monzingo, R.A., and Miller, T.W. (1980) Introduction to Adaptive Arrays. New York: Wiley, 1980.

[3] Hansen, P.M., and Loughlin, J.P. (1981) Adaptive array for elimination of multipath interference at HF.
IEEE Transactions on Antennas and Propagation, AP-29, 6 (Nov. 1981), 836-841.

[4] Shan, T.J., and Kailath, T. (1985).

Adaptive beamforming for coherent signals and interference. IEEE Transactions on Acoustics, Speech, and Signal Processing, ASSP-33, 3 (June 1985), 527-536.

[5] Dixon, R.C. (1984) Spread Spectrum Systems (2nd ed.) New York: Wiley, 1984.

[6] Ogawa, Y., Ohmiya, M., and Itoh, K. (1985) An LMS adaptive array using a pilot signal. IEEE Transactions on Aerospace and Electronic Systems, $A E S-21,6$ (Nov. 1985), 777-782.

[7] Widrow, B., Duvall, K., Gooch, R.P., and Newman, W.C. (1982)

Signal cancellation phenomena in adaptive antennas: causes and cures.

IEEE Transactions on Antennas and Propagation, AP-30, 3 (May 1982), 469-478.

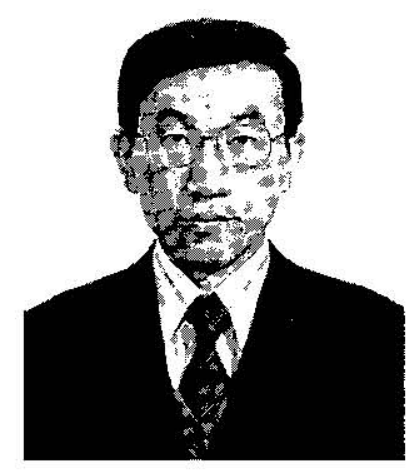

Yasutaka Ogawa (S'73-M'78) was born in Sapporo, Japan, on March 22, 1950. He received the B.S., M.S., and Ph.D. degrees from Hokkaido University, Sapporo, Japan, in 1973, 1975, and 1978, respectively.

Since 1979 , he has been with Hokkaido University, where he is presently an Associate Professor of Electronic Engineering. His special interests are in adaptive array antennas, frequency and time comparison using a broadcasting satellite, and digital communication systems.

Dr. Ogawa is a member of the Institute of Electronics and Communication Engineers of Japan.
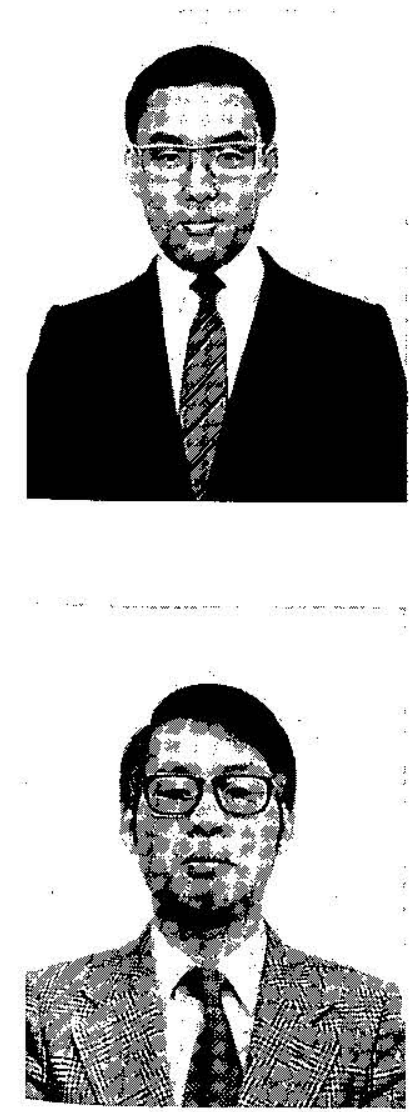

Manabu Ohmiya was born in Sapporo, Japan, on December 6, 1957. He received the B.S.E.E. and M.S. degrees from Hokkaido University, Sapporo, Japan, in 1981 and 1983 , respectively.

Since 1983, he has been with Hokkaido University, where he is presently an Instructor of Electronic Engineering. His special interests are in adaptive array antenna, signal processing, and optical communication.

Mr. Ohmiya is a member of the Institute of Electronics and Communication Engineers of Japan.

Kiyohiko Itoh (M'71) was born in Sapporo, Japan, on May 15, 1939. He received the B.S.E.E. degree in 1963, the M.S. degree in 1965, and Ph.D. degree in 1973 from Hokkaido University, Sapporo, Japan.

Since 1965 , he has been on the Faculty of Engineering at Hokkaido University, where he is a Professor of Electronic Engineering. During 1970-1971, he was with the Department of Electrical and Computer Engineering, Syracuse University, Syracuse, N.Y., as a Research Associate on leave from Hokkaido University. His special interests are in electromagnetic radiation, frequency and time comparison using a broadcasting satellite, wave optics, mobile radio communications, and solar power satellite.

Dr. Itoh is a member of the Institute of Electronics and Communication Engineers of Japan and also an international coordinator of the Antennas and Propagation Society of IEEE. 